\title{
Оптимизация режимов генерации волоконных лазеров на основе генетического алгоритма
}

\author{
Д.А. Художиткова ${ }^{1, *}$, А.Е. Беднякова ${ }^{1,2}$ \\ ${ }^{1}$ Новосибирский государственный университет \\ ${ }^{2}$ Институт вычислительных технологий СО РАН \\ "E-mail: ashulya-m@mail.ru
}

DOI:10.31868/RFL2018.148-149

Современная наука все чаще ставит оптимизационные задачи для сложных нелинейных многопараметрических систем. В данной работе такой системой является волоконный лазер. Исследуемый лазер представляет собой полностью волоконный кольцевой резонаор, состоящий из стандартного одномодового волокна, волокна, сохраняющего поляризацию, усиливающего волокна, фильтров и поляризационного светоделителя. Синхронизация мод достигается за счет эффекта нелинейного вращения поляризации [1].

В рамках работы было рассмотрено две математические модели: скалярная, в которой распространение сигнала по световоду рассчитывается с помощью обобщенного нелинейного уравнения Шредингера, и гибридная, в которой волокно, не сохраняющее поляризацию, моделировалась парой нелинейных уравнений Шредингера для двух компонент поляризации. Остальные устройства моделировались точечно.

Для решения оптимизационных задач хорошо зарекомендовал себя генетический алгоритм [2-3]. В скалярной модели оптимизация проводилась по трем параметрам: $L_{D S F}, L_{P M F}, P_{S A T}$ - длины стандартного одномодового и сохраняющего поляризацию волокон, а также мощность насыщения. В гибридной модели были добавлены еще два параметра $\chi, \psi$ - углы поворотов четвертьволновой и полуволновой пластин соответственно. Целью работы является поиск оптимальных параметров лазера для получения устойчивого одноимульсного режима генерации с максимальной энергией импульса. В качестве функции приспособленности выбрана энергия, если режим одноимпульсный и стабильный.

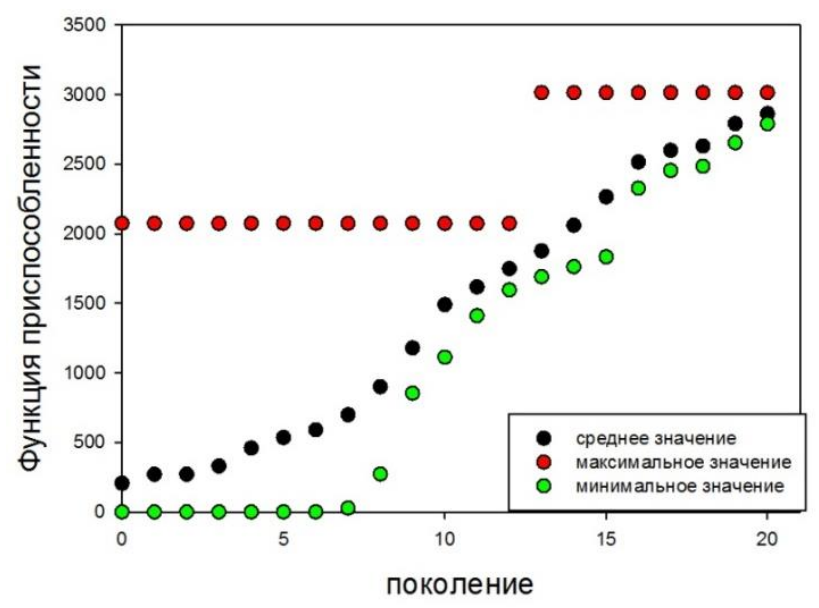

Рис.1. Сходимость генетического алгоритма 
Для численного решения обобщенного нелинейного уравнения Шредингера был применен метод расщепления по физическим процессам с использованием быстрого преобразования Фурье на линейном шаге в симметричной форме. Влияние на сигнал остальных устройств резонатора, таких как усилитель, фильтр, поляризатор, учитывается в расчетах точечно.

На рисунке 1 показана сходимость среднего и минимального значений целевой функции к найденному алгоритмом максимуму, что говорит о сходимости алгоритма.

На рисунке 2 представлена сходимость параметров оптимизации в течение работы алгоритма. Видно, что параметры локализованы в небольшой области пространства параметров в окрестности оптимума.
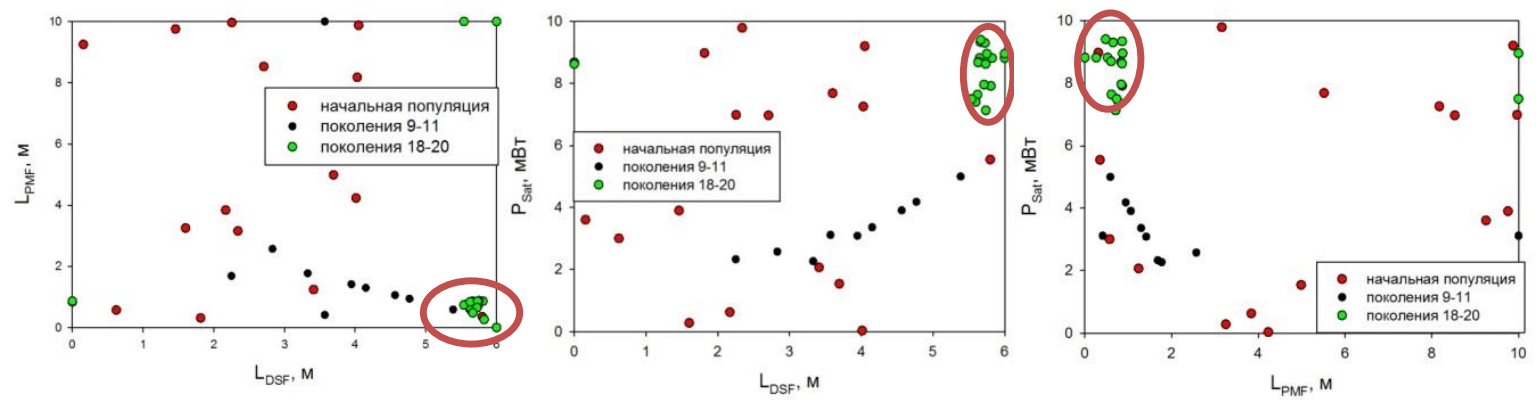

Рис.2. Сходимость оптимизационных параметров в процессе эволюции алгоритма

В рамках данной работы была написана программа на языке $\mathrm{C}++$, решающая поставленную оптимизационную задачу. Для реализации генетического алгоритма была использована библиотека GALib. Получены оптимальные значения параметров, при которых достигается одноимульсный режим генерации с наибольшей энергией. Благодаря генетическому алгоритму и распараллеливанию удалось значительно сократить время расчетов по сравнению с перебором параметров.

Исследование выполнено за счет гранта Российского научного фонда (проект № 17-72-30006)

\section{Литература}

[1] D. S. Kharenko, I. S. Zhdanov et al, Opt. Lett. 42, 3221-3224 (2017)

[2] X. Fu, J. N. Kutz, Optics Express 21, 6526-6537 (2013)

[3] U. Andral, R. Si Fodil et al, Optica 2, 275-278 (2015) 